\title{
IMPACTO DA DEPRESSÃO PÓS-PARTO NO ALEITAMENTO MATERNO: UMA REVISÃO INTEGRATIVA DE LITERATURA
}

\section{REVISÃO INTEGRATIVA}

SILVA, Victória Marques ${ }^{1}$, GREGÓRIO, Débora Maria Alves², SILVA, Larissa Matos ${ }^{3}$, SOUZA, Mariana Chastel de Liz ${ }^{4}$, PORFIRIO, Regiane Baptista Martins ${ }^{5}$

SILVA, Victória Marques. Et al. Impacto da depressão pós-parto no aleitamento materno: uma revisão integrativa de literatura. Revista Científica Multidisciplinar Núcleo do Conhecimento. Ano 06, Ed. 06, Vol. 10, pp. 60-74. Junho de 2021. ISSN: 2448-0959, Link de acesso: https://www.nucleodoconhecimento.com.br/saude/posparto-no-aleitamento, DOI: 10.32749/nucleodoconhecimento.com.br/saude/posparto-no-aleitamento

\section{RESUMO}

Objetivo: Trazer o conhecimento necessário para os profissionais de enfermagem sobre a importância em identificar gestantes com sinais e sintomas de depressão pósparto e como isso impacta no aleitamento materno exclusivo. Pergunta problema: Como a DPP pode afetar o aleitamento materno e a relação entre a mãe e o bebê? Metodologia: Estudo de revisão integrativa de literatura, exploratório realizado em 2020. Principais resultados: Com alguns estudos, foi possível perceber que pela falta de condições psicológicas, a relação entre mãe-bebê apresenta barreiras para ser

\footnotetext{
1 Graduação em Enfermagem.

2 Graduação em Enfermagem.

${ }^{3}$ Graduação em Enfermagem.

${ }^{4}$ Graduação em Enfermagem.

${ }^{5}$ Orientadora.
}

$\mathrm{RC}: 88818$

Disponível em: https://www.nucleodoconhecimento.com.br/saude/pos-parto-no-aleitamento 
desenvolvida e que a falta de aleitamento materno exclusivo aumenta em 15 vezes o risco de morte infantil quando comparamos a saúde dos bebês com AME.

Palavras-chave: Aleitamento, Depressão, Enfermagem, Saúde da Criança.

\section{INTRODUÇÃO}

A depressão maior é um transtorno mental que persiste por pelo menos duas semanas com episódios que apresentam tristeza profunda e a perda de interesse em atividades que antes despertavam prazer. Para a sustentação do diagnóstico, é necessário ter confirmada a presença de pelo menos outros quatro sintomas específicos, de acordo com o Manual Diagnóstico e Estatístico de Transtornos Mentais IV (DSM) (INSTITUTO MUNICIPAL DE INVESTIGACIÓN MÉDICA, 1995). É também considerada um problema de saúde pública, onde estima-se afetar mais de 300 milhões de pessoas no mundo, de qualquer faixa etária (ORGANIZAÇÃO MUNDIAL DA SAÚDE, 2018). Segundo a Organização Mundial da Saúde (OMS), na população brasileira, o predomínio é de $15,5 \%$ e somente na atenção primária apresenta uma prevalência de 10,4\%. (MINISTÉRIO DA SAÚDE, 2019)

As mulheres estão mais susceptíveis a desenvolver a depressão (ORGANIZAÇÃO MUNDIAL DA SAÚDE, 2018), e durante o período gravídico, o enfermeiro/a busca sinais e sintomas indicativos do diagnóstico de depressão logo no pré-natal, como forma de prevenção, pois trata-se de uma etapa delicada na vida da mulher que envolverá uma série de alterações fisiológicas e hormonais, além de outras mudanças que envolvem o âmbito emocional e racional, tornando-se um fator de risco para tal condição. (SOCIEDADE BENEFICENTE ISRAELITA BRASILEIRA ALBERT EINSTEIN, 2019)

Durante o período puerperal, o risco do desenvolvimento da Depressão Pós-parto (DPP) é alto pelo fato da mulher estar lidando com novas rotinas, além das modificações físicas e mentais. Os sintomas depressivos podem ser notados desde a

$\mathrm{RC}: 88818$

Disponível em: https://www.nucleodoconhecimento.com.br/saude/pos-parto-no-aleitamento 
primeira semana ou até três meses após o parto e podem ser similares aos da depressão maior, somando ainda, o comprometimento do vínculo entre mãe-filho. (LINO et al., 2020) (ABUCHAIM et al., 2016)

Segundo a Teoria da Autoeficácia, o comportamento de um indivíduo é moldado conforme este interpreta seus sentimentos, logo, a DPP influencia negativamente todas as ações maternas (ABUCHAIM et al., 2016). Segundo Vieira et al., (2018) mulheres com baixa autoeficácia apresentam três vezes mais chances de interromper o aleitamento materno precocemente.

Mulheres que apresentaram depressão na gestação e no pós-parto têm grande dificuldade em criar o vínculo mãe-filho e pelo sentimento de insuficiência ou culpa, não se sentem capazes de cuidar de seus filhos. Crianças que são expostas a quadros depressivos acabam tendo prejuízos em relação ao desenvolvimento cognitivo e motor, mudanças de temperamento repentino e, em seu estado nutricional pela falta de amamentação materna exclusiva (AME) até o sexto mês de vida. (LINO et al., 2020)

O leite materno é crucial nos primeiros meses de vida pois, do ponto de vista nutricional é a partir deste alimento que o bebê terá o fortalecimento de seu sistema imunológico e terá um melhor desenvolvimento. $\mathrm{O}$ aleitamento também traz inúmeros benefícios à mãe, pois com a lactação, os níveis de estresse são diminuídos e consequentemente reduz a sintomatologia depressiva. (LINO et al., 2020)

A motivação das autoras para o desenvolvimento desse estudo é mostrar o quanto é necessária e importante a prestação da assistência humanizada à mulher desde o pré-natal até o pós-parto, se tornando indispensável o cuidado da enfermagem e a detecção de possíveis repercussões prejudiciais futuras no aleitamento materno e na relação do binômio mãe-filho. Então, a pergunta deste estudo é: Como a DPP pode afetar $\mathrm{o}$ aleitamento materno e a relação entre a mãe e o bebê?

$\mathrm{RC}: 88818$

Disponível em: https://www.nucleodoconhecimento.com.br/saude/pos-parto-no-aleitamento 
O objetivo deste estudo é realizar uma revisão de literatura com o intuito de ampliar o conhecimento dos profissionais de enfermagem quanto à importância da identificação das gestantes com fatores de riscos de depressão pós-parto e sobre os impactos dela no aleitamento materno.

\section{METODOLOGIA}

Este artigo foi elaborado pelo método de revisão integrativa de literatura, que trata da construção de um texto que tem como objetivo colocar de forma simples e objetiva os resultados obtidos a partir de pesquisas sobre um único assunto de forma sistemática, ordenada e abrangente, disponibilizando muitas informações amplas, formando assim um corpo de conhecimento. (HASSAN et al., 2016)

Para o desenvolvimento deste artigo, foram realizadas pesquisas durante o mês de outubro do ano de 2020, utilizando os descritores pesquisados na plataforma dos Descritores de Ciências da Saúde (DeCS): Aleitamento; Depressão; Enfermagem e Saúde da Criança.

As bases de dados utilizadas foram: Biblioteca Virtual em Saúde (BIREME), Literatura Latino Americana e do Caribe em Ciências da Saúde (LILACS), Medline Literatura Internacional em Ciências da Saúde (MEDLINE), Scientific Eletronic Library Online (ScieLO), Google Acadêmico, Direct Science e o website oficial da Organização Mundial da Saúde (OMS).

Como critérios de inclusão foram utilizados os idiomas português e inglês, período de 2016 a 2020, com pelo menos dois dos descritores selecionados, texto compatível ao tema, sendo excluídos os artigos que não atendiam a ideia principal desse artigo.

Por se tratar de estudo bibliográfico não foi necessária a aprovação do Comitê de Ética em Pesquisa. Também não há conflito de interesse entre as autoras envolvidas.

$\mathrm{RC}: 88818$

Disponível em: https://www.nucleodoconhecimento.com.br/saude/pos-parto-no-aleitamento 


\section{RESULTADOS}

Este artigo trata-se de uma revisão integrativa da literatura, utilizando como critérios de inclusão: textos em português e inglês, entre os anos de 2016-2020, tema relacionado à intenção da pesquisa e os descritores selecionados pelas autoras.

Após adicionar os critérios pré-estabelecidos foram encontrados sete artigos na base de dados SciELO, seis na BVS, dois no Google Acadêmico e um na Direct Science. Após leitura dos artigos na íntegra, destacaram-se 13 estudos, os quais estão detalhados no quadro 1.

Quanto às categorias dos estudos encontrados, 30,77\% abordam discussões focadas na "relação da DPP e aleitamento materno", 23,08\% no "aleitamento materno", 15,38\% "depressão na gestação" e "Atenção Primária em Saúde no puerpério", e $7,69 \%$ "maternidade" e "nutrição infantil".

No quadro 1 encontra-se a síntese dos artigos levantados por meio dos critérios de inclusão e exclusão.

Quadro 1: Características das publicações utilizadas nesse estudo.

\begin{tabular}{|c|c|c|c|c|}
\hline Autoria & $\begin{array}{l}\text { Ano de } \\
\text { publicação }\end{array}$ & Periódico & Título do estudo & Objetivo do estudo \\
\hline $\begin{array}{l}\text { Silva e } \\
\text { col. }\end{array}$ & 2017 & $\begin{array}{l}\text { Jornal de } \\
\text { Pediatria } \\
\text { (Rio J.) }\end{array}$ & $\begin{array}{l}\text { Associação entre } \\
\text { depressão pós- } \\
\text { parto e prática do } \\
\text { aleitamento } \\
\text { materno exclusivo } \\
\text { nos primeiros três } \\
\text { meses de vida. }\end{array}$ & $\begin{array}{l}\text { Verificar a associação } \\
\text { entre a depressão } \\
\text { pós-parto e a } \\
\text { ocorrência } \\
\text { aleitamento } \\
\text { exclusivo. }\end{array}$ \\
\hline
\end{tabular}

$\mathrm{RC}: 88818$

Disponível em: https://www.nucleodoconhecimento.com.br/saude/pos-parto-no-aleitamento 


\begin{tabular}{|c|c|c|c|c|}
\hline $\begin{array}{l}\text { Moraes e } \\
\text { col. }\end{array}$ & 2020 & $\begin{array}{l}\text { Revista de } \\
\text { Enfermagem } \\
\text { Referência }\end{array}$ & $\begin{array}{l}\text { Percepção sobre } \\
\text { a importância do } \\
\text { aleitamento } \\
\text { materno pelas } \\
\text { mães e } \\
\text { dificuldades } \\
\text { enfrentadas no } \\
\text { processo de } \\
\text { amamentação. }\end{array}$ & $\begin{array}{l}\text { Conhecer a } \\
\text { percepção das mães } \\
\text { sobre a importância } \\
\text { do AM e identificar as } \\
\text { principais } \\
\text { dificuldades } \\
\text { enfrentadas para a } \\
\text { prática do AM e/ou da } \\
\text { sua manutenção. }\end{array}$ \\
\hline $\begin{array}{l}\text { Oliveira M } \\
\text { e col. }\end{array}$ & 2019 & $\begin{array}{l}\text { Enfermagem } \\
\text { foco }\end{array}$ & $\begin{array}{l}\text { Sentimento de } \\
\text { mulheres com } \\
\text { depressão pós- } \\
\text { parto frente ao } \\
\text { aleitamento } \\
\text { materno. }\end{array}$ & $\begin{array}{l}\text { Descrever } \\
\text { sentimentos de } \\
\text { mulheres com } \\
\text { depressão pós-parto } \\
\text { frente ao aleitamento } \\
\text { materno. }\end{array}$ \\
\hline $\begin{array}{l}\text { Vieira } \\
\text { col. }\end{array}$ & 2018 & $\begin{array}{l}\text { Revista } \\
\text { Latino- } \\
\text { Americana } \\
\text { de } \\
\text { Enfermagem }\end{array}$ & $\begin{array}{l}\text { Autoeficácia para } \\
\text { amamentação e } \\
\text { depressão pós- } \\
\text { parto: estudo de } \\
\text { coorte. }\end{array}$ & $\begin{array}{l}\text { Avaliar a autoeficácia } \\
\text { para amamentação, a } \\
\text { presença de sintomas } \\
\text { de depressão no } \\
\text { período pós-parto e a } \\
\text { associação entre } \\
\text { autoeficácia na } \\
\text { amamentação } \\
\text { depressão pós-parto, } \\
\text { com a interrupção do } \\
\text { aleitamento materno } \\
\text { exclusivo. }\end{array}$ \\
\hline
\end{tabular}

$\mathrm{RC}: 88818$

Disponível em: https://www.nucleodoconhecimento.com.br/saude/pos-parto-no-aleitamento 


\begin{tabular}{|c|c|c|c|c|}
\hline $\begin{array}{l}\text { Abuchaim } \\
\text { e col. }\end{array}$ & 2016 & $\begin{array}{l}\text { Acta Paulista } \\
\text { de } \\
\text { Enfermagem }\end{array}$ & $\begin{array}{l}\text { Depressão pós- } \\
\text { parto } \\
\text { autoeficácia } \\
\text { materna para } \\
\text { amamentar: } \\
\text { prevalência } \\
\text { associação. }\end{array}$ & $\begin{array}{lr}\text { Identificar } & \text { a } \\
\text { prevalência de } & \text { de } \\
\text { sintomas } & \text { pós-parto } \\
\text { depressão } & \text { para } \\
\text { e o ní de } \\
\text { autoeficácia de } \\
\text { amamentar, entre } \\
\text { puérperas atendidas } \\
\text { num Centro de } \\
\text { Incentivo aleitamento Materno, } \\
\text { e analisar possíveis } \\
\text { associações. }\end{array}$ \\
\hline $\begin{array}{l}\text { Baratieri e } \\
\text { col. }\end{array}$ & 2019 & $\begin{array}{l}\text { Ciência } \\
\text { Saúde } \\
\text { Coletiva }\end{array}$ & $\begin{array}{l}\text { Ações do } \\
\text { programa de } \\
\text { puerpério na } \\
\text { atenção primária: } \\
\text { uma revisão } \\
\text { integrativa. }\end{array}$ & $\begin{array}{l}\text { Sistematizar } \\
\text { conhecimento } \\
\text { produzido sobre as } \\
\text { ações de programas } \\
\text { de atenção pós-parto } \\
\text { no âmbito da APS, } \\
\text { tanto em nível } \\
\text { nacional, como } \\
\text { internacional. }\end{array}$ \\
\hline $\begin{array}{l}\text { Giordani } \\
\text { e col. }\end{array}$ & 2018 & $\begin{array}{l}\text { Ciência } \\
\text { Saúde } \\
\text { Coletiva }\end{array}$ & $\begin{array}{l}\text { Maternidade } \\
\text { amamentação: } \\
\text { identidade, corpo } \\
\text { e gênero. }\end{array}$ & $\begin{array}{l}\text { Propõe uma reflexão } \\
\text { em torno da } \\
\text { maternidade, } \\
\text { problematizando a } \\
\text { experiência } \\
\text { amamentação e a } \\
\text { construção } \\
\text { identidade, de }\end{array}$ \\
\hline
\end{tabular}

RC: 88818

Disponível em: https://www.nucleodoconhecimento.com.br/saude/pos-parto-no-aleitamento 


\begin{tabular}{|c|c|c|c|c|c|}
\hline & & & & & $\begin{array}{l}\text { aproximando de } \\
\text { conceitos } \\
\text { sociológicos do } \\
\text { interacionismo de } \\
\text { Anselm Levi Strauss. }\end{array}$ \\
\hline $\begin{array}{l}\text { Costa } \\
\text { col. }\end{array}$ & e & 2018 & $\begin{array}{l}\text { Ciência e } \\
\text { Saúde } \\
\text { Coletiva }\end{array}$ & $\begin{array}{l}\text { Transtornos } \\
\text { mentais na } \\
\text { gravidez e } \\
\text { condições do } \\
\text { recém-nascido: } \\
\text { estudo } \\
\text { longitudinal com } \\
\text { gestantes } \\
\text { assistidas na } \\
\text { atenção básica. }\end{array}$ & $\begin{array}{l}\text { Verificar a presença e } \\
\text { a associação entre } \\
\text { diagnósticos } \\
\text { prováveis de } \\
\text { transtornos mentais } \\
\text { em gestantes da } \\
\text { atenção básica e } \\
\text { condições dos recém- } \\
\text { nascidos. }\end{array}$ \\
\hline $\begin{array}{l}\text { Lima } \\
\text { col. }\end{array}$ & e & 2017 & $\begin{array}{l}\text { Acta Paulista } \\
\text { de } \\
\text { Enfermagem }\end{array}$ & $\begin{array}{l}\text { Sintomas } \\
\text { depressivos na } \\
\text { gestação } \\
\text { fatores } \\
\text { associados: } \\
\text { estudo } \\
\text { longitudinal. }\end{array}$ & $\begin{array}{l}\text { Identificar a } \\
\text { frequência de } \\
\text { sintomas depressivos } \\
\text { no decorrer da } \\
\text { gestação e verificar } \\
\text { sua associação com } \\
\text { variáveis } \\
\text { sociodemográficas, } \\
\text { obstétricas e de } \\
\text { saúde. }\end{array}$ \\
\hline $\begin{array}{l}\text { Silva } \\
\text { col. }\end{array}$ & e & 2020 & $\begin{array}{l}\text { Revista de } \\
\text { Enfermagem } \\
\text { UFPE }\end{array}$ & $\begin{array}{lr}\text { Intervenções } & \text { do } \\
\text { enfermeiro } & \text { na } \\
\text { atenção } & \text { e } \\
\text { prevenção } & \text { da }\end{array}$ & $\begin{array}{l}\text { Identificar a produção } \\
\text { científica sobre as } \\
\text { ações/intervenções } \\
\text { que podem ser } \\
\text { desenvolvidas pelo }\end{array}$ \\
\hline
\end{tabular}

$\mathrm{RC}: 88818$

Disponível em: https://www.nucleodoconhecimento.com.br/saude/pos-parto-no-aleitamento 


\begin{tabular}{|c|c|c|c|c|}
\hline & & & $\begin{array}{l}\text { depressão } \\
\text { puerperal. }\end{array}$ & $\begin{array}{l}\text { enfermeiro na } \\
\text { atenção e prevenção } \\
\text { de danos da } \\
\text { depressão puerperal. }\end{array}$ \\
\hline Lino e col. & 2020 & $\begin{array}{l}\text { Revista } \\
\text { Nursing }\end{array}$ & $\begin{array}{l}\text { O impacto da } \\
\text { depressão pós- } \\
\text { parto no } \\
\text { aleitamento } \\
\text { materno e no } \\
\text { desenvolvimento } \\
\text { infantil: Uma } \\
\text { revisão } \\
\text { integrativa. }\end{array}$ & $\begin{array}{l}\text { Identificar o impacto } \\
\text { da depressão pós- } \\
\text { parto no aleitamento } \\
\text { materno e no } \\
\text { desenvolvimento } \\
\text { infantil. }\end{array}$ \\
\hline $\begin{array}{l}\text { Hassan e } \\
\text { col. }\end{array}$ & 2016 & $\begin{array}{l}\text { Revista de } \\
\text { Saúde } \\
\text { Pública }\end{array}$ & $\begin{array}{l}\text { Saúde mental } \\
\text { materna e estado } \\
\text { nutricional de } \\
\text { crianças aos seis } \\
\text { meses de vida. }\end{array}$ & $\begin{array}{l}\text { Analisar se saúde } \\
\text { mental materna } \\
\text { associa-se ao estado } \\
\text { nutricional infantil no } \\
\text { sexto mês de vida. }\end{array}$ \\
\hline $\begin{array}{l}\text { Menezes } \\
\text { e col. }\end{array}$ & 2019 & BIUS & $\begin{array}{l}\text { A importância da } \\
\text { amamentação na } \\
\text { formação de } \\
\text { vínculos afetivos } \\
\text { saudáveis entre } \\
\text { mamãe/bebê. }\end{array}$ & $\begin{array}{lr}\text { Analisar se } & \text { a } \\
\text { amamentação } & \\
\text { interfere } & \\
\text { positivamente } & \text { na } \\
\text { constituição } & \text { de } \\
\text { vínculos afetivos } \\
\text { saudáveis } \\
\text { mamãe/bebê. }\end{array}$ \\
\hline
\end{tabular}

Fonte: Autoria própria (2020)

$\mathrm{RC}: 88818$

Disponível em: https://www.nucleodoconhecimento.com.br/saude/pos-parto-no-aleitamento 


\section{DISCUSSÃO}

Para a mulher, a gestação é marcada por mudanças constantes e de muitas transformações em seu organismo e nos setores sociopsicológicos, onde o maior desafio é saber conviver com essa nova experiência que muitas vezes não foi planejada, onde a mulher não se encontra preparada para esse acontecimento tão importante. "Dentre as manifestações psíquicas, a mais comum no período do puerpério são: baby blues ou tristeza materna, a depressão pós-parto (DPP) e a psicose puerperal." A DPP aparece como a mais frequente e é a que causa um maior impacto tanto para a mãe quanto para o bebê que acabou de nascer. (DE OLIVEIRA et al., 2019)

De acordo com Giordani et al. (2018), a identidade feminina sofre diversas alterações após o nascimento de um filho, pois além das mudanças psicológicas e fisiológicas, também é chegada a responsabilidade. Com isso, vemos transformações em seus relacionamentos pessoais e interpessoais. Os autores citados, afirmam que "a mulher passa a assumir uma nova condição de si, da vida, das relações, dos outros, provocando uma transformação de comportamento, posturas e, finalmente, uma mudança completa na sua autoimagem".

Para mulheres que estão completamente saudáveis, a amamentação já é um momento considerado delicado em decorrência de todos os riscos de complicação que estão associados apesar da técnica correta.

Em um estudo realizado por De Oliveira et al., (2019), com 20 mulheres que têm ou tiveram depressão pós-parto, houve uma entrevista na qual elas relataram como foi a experiência do transtorno psicológico diagnosticado com o aleitamento materno. Percebeu-se que a relação entre mãe e filho apresentava barreiras devido à falta de condições psicológicas com falas bem impactantes.

$\mathrm{RC}: 88818$

Disponível em: https://www.nucleodoconhecimento.com.br/saude/pos-parto-no-aleitamento 
"Mal consegui amamentar meu filho, foi horrível eu tinha uma sensação de abuso, não tinha vontade de dar o peito à criança" (Violeta): "Foi ruim, sentia a obrigação de amamentar, não era feliz amamentando" (Orquídea): "A criança não pegava o peito e isso me estressava" (Gloriosa): "Era estranho, não sentia afeto pelo bebê" (Jasmim): "Me sentia forçada a amamentar, não tinha desejo de amamentar" (Antúrio): "Tinha um sentimento de medo acompanhado de tristeza, receio para amamentar" (Cerejeira): "Quando colocava o bebê no peito sentia dor e desconforto, toda vez que eu lembrava que tinha que amamentar ficava mais triste" (Lírio). (DE OLIVEIRA et al., 2019, p. 90)

A partir destes relatos levantados, percebeu-se que o vínculo materno é prejudicado pela depressão pós-parto, uma vez que o aleitamento é responsável por fortalecer este binômio e é tratado com rejeição e medo quando ela está nestas condições. A ocitocina é liberada quando o bebê recebe um ato de afeto e quando isso não ocorre de forma esperada, ele deixa de sentir de forma proporcional a sensação de segurança e carinho. (de OLIVEIRA et al., 2019) (COELHO, et al., 2019)

Um estudo realizado por Hassan, Werneck e Hasselmann (2016) tem como intuito analisar a saúde mental de mulheres puérperas e relacioná-las ao estado nutricional de seus filhos até o sexto mês de vida. Foram tiradas médias de peso, altura e comprimento das crianças, ${ }_{1}$ e expressas em escores considerando as curvas do Word Health Organization (WHO), 2006. A saúde mental das mulheres foi aferida pelo General Health Questionnaire e tendo em vista todos os resultados levantados, foi obtido um número expressivo de mulheres que estavam com a saúde mental comprometida por depressão e, consequentemente, seus filhos apresentaram níveis abaixo do esperado em relação à peso, altura e comprimento. Assim foi constatado que a qualidade da saúde mental da mãe está relacionada ao estado nutricional do bebê de forma positiva e negativamente.

Um artigo publicado em setembro por Silva et al., (2017), na Espanha, mostra como o estresse, a depressão pós-parto e o otimismo deposicional influenciam na adesão

$\mathrm{RC}: 88818$

Disponível em: https://www.nucleodoconhecimento.com.br/saude/pos-parto-no-aleitamento 
do $\mathrm{AME}$, além de destacar que as questões socioculturais, trabalho e família são fatores importantes. Demonstra também, que os fatores psicológicos foram pouco explorados durante a lactação, mostrando a importância de levar em consideração esses fatores para ajudar a desenvolver um programa de intervenção na amamentação eficaz.

$\mathrm{Na}$ Europa, os países têm licença-maternidade com diferentes durações, onde em alguns países, acaba sendo de apenas três meses, época mais comum para o início de conflitos trabalho-família, associando o estresse da mãe e a amamentação materna exclusiva, resultando em um dos principais motivos da sua interrupção. Entre outros motivos, estão também: alta hospitalar precoce, mastite, deficiência do crescimento infantil, baixa saúde mental ou emocional, depressão pós-parto, baixo apoio e aconselhamento sobre amamentação. (SILVA et al., 2017)

O estudo nos mostra também que existe um risco 15 vezes maior de morte infantil em crianças sem a amamentação materna exclusiva, quando comparada aos bebês com AME. Além de trazer que as taxas de amamentação em todo o mundo são inferiores às recomendadas e que vão diminuindo durante os primeiros seis meses após o parto. No primeiro mês, as taxas de AME variam entre $68 \%$ e $84 \%$, já no sexto mês após o parto, apenas $13-20 \%$ das mulheres permanecem na AME. A Região Europeia é a que apresenta as taxas mais baixas, na Espanha, apenas 66\% dos bebês são amamentados exclusivamente no primeiro mês, tendo uma queda para $28 \%$ no sexto mês. (SILVA et al., 2017)

Em pesquisas realizadas para rastreamento da sintomatologia depressiva na gestação, foi identificado que mulheres grávidas em idades precoces (menores de 18 anos), de baixa renda, sem apoio ou vínculo familiar, maiores de 40 anos, baixa escolaridade, usuárias de drogas ilícitas, tabagistas, etilistas, primigestas, mulheres que já sofreram algum tipo de violência física ou psicológica e as de gravidez não planejada ou indesejada estão mais suscetíveis a apresentarem sintomas depressivos em qualquer período da gestação (LIMA et al., 2017).

$\mathrm{RC}: 88818$

Disponível em: https://www.nucleodoconhecimento.com.br/saude/pos-parto-no-aleitamento 
O aleitamento interrompido precocemente traz consigo inúmeros danos, em sua maioria negativos para a mãe e bebê. A mulher deixa de estimular a produção de ocitocina que aconteceria durantes as mamadas e então consequentemente ${ }_{2}$ diminui sua ação benéfica de involução uterina, perda de peso, redução de hemorragia pósparto e diminuição do risco de cancro de mama e colo de útero. (MORAES et al., 2020)

Sabe-se que o leite materno contém todos os nutrientes que o recém-nascido (RN) necessita para um desenvolvimento cognitivo e motor saudável, uma vez que é rico em proteínas, óleos, vitaminas, gorduras e ácidos gordos. Os benefícios vão desde a prevenção da mortalidade infantil até infecções, diabetes e obesidade (MORAES et al., 2020). Entretanto, para Vieira et al., (2018) "mulheres com sintomas depressivos durante a gestação apresentam maior probabilidade de introduzir, precocemente, fórmula láctea na alimentação da criança".

Segundo Costa et al., (2018) a investigação de sintomas depressivos na atenção básica ainda é um desafio para os profissionais de saúde, com dificuldades inclusive na elaboração dos diagnósticos. O pré-natal é realizado na atenção básica, onde é feito o devido acompanhamento fisiológico e psicológico da mulher e do desenvolvimento fetal. Também é feita a estimulação da amamentação antes mesmo do nascimento do bebê, devido aos seus inúmeros benefícios.

A atenção primária em saúde (APS) é a principal quando se trata dos cuidados com a mulher no pós-parto. É durante as consultas deste período que ocorre a prevenção, detecção precoce e tratamento de complicações, ou seja, a qualificação dos profissionais é fundamental para a diminuição de agravos. Quando se trata do aleitamento materno, a APS possui um foco maior para o bebê, sendo insuficiente quanto ao acolhimento da mulher e as dificuldades que ela possa estar enfrentando. (BARATIERI e NATAL, 2019)

É de suma importância que a enfermagem tenha conhecimento sobre a depressão puerperal, pois podem intervir de diversas maneiras, desde as consultas do pré-natal

$\mathrm{RC}: 88818$

Disponível em: https://www.nucleodoconhecimento.com.br/saude/pos-parto-no-aleitamento 
com investigações de conflitos pessoais, de sinais e sintomas de uma possível DPP, ou até mesmo após o parto, realizando uma visita domiciliar proporcionando promoção à saúde e o acolhimento necessários para a mãe e o bebê.

Verifica-se que a consulta e a visita domiciliar são momentos oportunos para o acolhimento e a escuta qualificada, visando um cuidado integral, dando liberdade para que sejam sanadas as dúvidas e, consequentemente, estimulando o autocuidado e 0 cuidado com a criança. (SILVA et al., 2020)

\section{CONCLUSÃO}

Percebe-se que para as mulheres diagnosticadas com DPP, o aleitamento materno é uma fase difícil, acompanhada de várias sensações negativas como tristeza, medo e desesperança.

Quando a mãe amamenta seu filho, proporciona a ele a sensação de conforto, segurança e carinho ${ }_{1}$ porém quando o AME é interrompido, o bebê pode ter mudanças de comportamento repentino como sono irregular e irritação, além de ter um atraso em seu desenvolvimento e até falhas no sistema imune.

A probabilidade da substituição do leite materno por fórmula láctea é considerada quando a mãe apresenta sintomas depressivos, sendo um fator que deve ser considerado com atenção por todos os profissionais que acompanham a saúde da criança lesada nutricionalmente.

Neste estudo foram abordados os impactos da depressão pós-parto no aleitamento materno, mostrando a importância das intervenções de enfermagem diante ao prénatal, sobre a saúde mental da puérpera, o quanto é significativo o olhar cuidadoso e o acolhimento do profissional da enfermagem. Sendo assim, implementando ações para que o bebê tenha um bom desenvolvimento e a relação mãe-filho seja saudável, onde esse vínculo é diretamente afetado pela presença da DPP, pois com ela muitas

$\mathrm{RC}: 88818$

Disponível em: https://www.nucleodoconhecimento.com.br/saude/pos-parto-no-aleitamento 
mulheres perdem ou não tem afeto pelo filho, tendo constante sentimento de insegurança e incerteza.

Observou-se, que existe a necessidade de mais pesquisas em campo sobre a DPP e sobre as intervenções da enfermagem diante ao assunto, pois encontramos um número maior de revisão integrativa de literatura, o que limita o profissional da saúde de ter mais conhecimentos para promoção de uma assistência adequada.

\section{REFERÊNCIAS}

ABUCHAIM, E. de S. V. et al. Depressão pós-parto e autoeficácia materna para amamentar: prevalência e associação. Acta paul. enferm., São Paulo, v. 29, n. 6, p. 664-670, dez. 2016. Disponível em: $<$ http://www.scielo.br/scielo.php?script=sci_arttext\&pid=S010321002016000600664\&Ing=en\&nrm=iso>. Acesso em: 03 dez. 2020. doi: http://dx.doi.org/10.1590/1982-0194201600093.

BARATIERI, T.; NATAL, S. Ações do programa de puerpério na atenção primária: uma revisão integrativa. Ciênc. saúde coletiva, Rio de Janeiro, v. 24, n. 11, p. 42274238 , nov. 2019.

$<$ http://www.scielo.br/scielo.php?script=sci_arttext\&pid=S1413-

81232019001104227\&Ing=en\&nrm=iso >. Acesso em: 03 dez. 2020. Epub out. 28, 2019. https://doi.org/10.1590/1413-812320182411.28112017.

COELHO, A.; et al. A importância da amamentação na formação de vínculos afetivos saudáveis entre mamãe/bebê. Boletim Informativo Unimotrisaúde em Sociogerontologia Online. 2019; 12(5). Disponível em: https://www.periodicos.ufam.edu.br/index.php/BIUS/article/view/6191.

COSTA, D. O.; et al. Transtornos mentais na gravidez e condições do recémnascido: estudo longitudinal com gestantes assistidas na atenção básica. Ciênc. saúde coletiva, Rio de Janeiro, v. 23, n. 3, p. 691-700, Mar. 2018. Disponível em: RC: 88818

Disponível em: https://www.nucleodoconhecimento.com.br/saude/pos-parto-no-aleitamento 
<http://www.scielo.br/scielo.php?script=sci_arttext\&pid=S1413-

$81232018000300691 \& \operatorname{lng}=$ en\&nrm $=$ iso $>$.

Acesso

em:

dez. 2020. https://doi.org/10.1590/1413-81232018233.27772015.

DE OLIVEIRA, M. G. et al. Sentimentos de mulheres com depressão pós-parto frente ao aleitamento materno. Enfermagem em Foco, [S.I.], v. 10, n. 3, nov. 2019. ISSN 2357-707X.

Disponível

em:

<http://revista.cofen.gov.br/index.php/enfermagem/article/view/1702/595>. Acesso em: 03 dez. 2020. doi: https://doi.org/10.21675/2357-707X.2019.v10.n3.1702.

GIORDANI, R. C. F. et al. Maternidade e amamentação: identidade, corpo e gênero. Ciênc. saúde coletiva, Rio de Janeiro, v. 23, n. 8, p. 2731-2739, Aug. 2018. Disponível em: <http://www.scielo.br/scielo.php?script=sci_arttext\&pid=S1413$81232018000802731 \& \operatorname{lng}=e n \& n r m=i s o>$. Acesso em: dez. 2020. https://doi.org/10.1590/1413-81232018238.14612016.

HASSAN, B. K.; et al. Maternal mental health and nutritional status of six-monthold infants. Rev. Saúde Pública, São Paulo, v. 50, 7, 2016. Disponível em: $<$ http://www.scielo.br/scielo.php?script=sci_arttext\&pid=S0034-

89102016000100206\&lng=en\&nrm=iso>. Acesso em: 03 dez. 2020. Epub Mar 22, 2016. http://dx.doi.org/10.1590/S1518-8787.2016050006237.

INSTITUTO MUNICIPAL DE INVESTIGACIÓN MÉDICA. DSM-IV. Manual diagnóstico y estadístico de los trastornos mentales. 1995. Disponível em: $<$ https://www.eafit.edu.co/ninos/reddelaspreguntas/Documents/dsm-iv-manualdiagnostico-estadistico-trastornos-mentales.pdf>. Acesso em: 29 de setembro.

LIMA, M. de O. P. et al. Sintomas depressivos na gestação e fatores associados: estudo longitudinal. Acta paul. enferm., São Paulo, v. 30, n. 1, p. 39-46, jan. 2017.2 Disponível em: <http://www.scielo.br/scielo.php?script=sci_arttext\&pid=S0103- 
$21002017000100039 \& \operatorname{lng}=$ en $\& n r m=i s o>$.

Acesso

em:

dez. 2020. https://doi.org/10.1590/1982-0194201700007.

LINO, C.; et al. O impacto da depressão pós-parto no aleitamento materno e no desenvolvimento infantil: Uma revisão integrativa. Nursing, São Paulo. 2020.

MINISTÉRIO DA SAÚDE. Depressão: causas, sintomas, tratamentos, diagnóstico e prevenção. 21 de agosto de 2019. Disponível em: <https://antigo.saude.gov.br/saude-de-a-z/depressao>. Acesso em: 29 de setembro.

MORAES, I. C. de; et al. Percepção sobre a importância do aleitamento materno pelas mães e dificuldades enfrentadas no processo de amamentação. Rev. Enf. Ref., Coimbra, v. serV, n. 2, p. e 19065, abr. 2020. Disponível em: $<$ http://www.scielo.mec.pt/scielo.php?script=sci_arttext\&pid=S0874-

02832020000200009\&lng=pt\&nrm=iso $>$.

Acesso

em:

03

dez. 2020. http://dx.doi.org/10.12707/RIV19065.

ORGANIZAÇÃO MUNDIAL DA SAÚDE. Depressão. Mar. de 2018. (Folha informativa). Disponível em: <https://www.paho.org/pt/topicos/depressao>. Acesso em: 29 de set.

SILVA, C. S.; et al. Association between postpartum depression and the practice of exclusive breastfeeding in the first three months of life, J. Pediatr. Rio J. Porto Alegre, v. 93, n. 4, p. 356-364, ago. 2017. Disponível em: <http://www.scielo.br/scielo.php?script=sci_arttext\&pid=S0021-

75572017000400356\&lng=en\&nrm=iso>. Acesso em: 03 dez. 2020. https://doi.org/10.1016/j.jped.2016.08.005.

SILVA, J. F. da; et al. Intervenções do enfermeiro na atenção e prevenção da depressão puerperal. Revista de Enfermagem UFPE online, [S.I.], v. 14, jul. 2020. ISSN 1981-8963.

Disponível em: 
<https://periodicos.ufpe.br/revistas/revistaenfermagem/article/view/245024/35555>. Acesso em: 03 dez. 2020. doi:https://doi.org/10.5205/1981-8963.2020.245024.

GOMES, M. N. de A.; SANTOS L. K. de O. Saúde da Mulher na Gestação, Parto e Puerpério. São Paulo: Hospital Israelita Albert Einstein: Ministério da Saúde, 2019. 56

p.: il.

Disponível

em:

$<$ https://atencaobasica.saude.rs.gov.br/upload/arquivos/202001/03091259-ntgestante-planificasus.pdf>. Acesso em: 5 de outubro.

VIEIRA, E. de S.; et al. Breastfeeding self-efficacy and postpartum depression: a cohort study. Rev. Latino-Am. Enfermagem, Ribeirão Preto, v. 26, e3035, 2018.

Disponível

em:

$<$ http://www.scielo.br/scielo.php?script=sci_arttext\&pid=S0104-

11692018000100348\&Ing=en\&nrm=iso>. Acesso em: 03 Dec. 2020. Epub set. 06, 2018. https://doi.org/10.1590/1518-8345.2110.3035.

Enviado: Dezembro, 2020.

Aprovado: Junho, 2021. 